attachments, as suggested by Lenuander, and to reference to peripheral structures, in accordance with the views of Hilton, Lange, Ross, Head, and Mackenzie. But it is generally possible to analyse the sensory symptoms in such a manner that the presence of true visceral pain can be recognised at some stage in the disease. In many conditions, such as the common forms of colic, visceral pain is present alone. On the other hand, referred pain, which is a result of the arrival in the spinal cord of impulses from an internal organ, is comparatively rarely present alone, as the impulses from the internal organ generally themselves give rise to visceral pain. Lastly, pain originating in the peritoneum in con. nexion with diseases of the alimentary canal is not uncommon in the absence of visceral pain.

Even when the adeguate stimulus is employed the viscera are very much less sensitive than the skin to painful stimulation. This is doubtless due, as Langley ${ }^{20}$ has pointed out, to the small number of afferent nerve fibres with which they are supplied, the total number distributed to the whole of the viscera of the cat being about the same as the number present in a single posterior spinal root.

One of the arguments most frequently used by those who do not believe that pain can originate in the viscera themselves is its inaccurate localisation. But seeing that painful stimuli cannot be accurately localised in the skin of such parts as the back of the trunk, it is not surprising that localisation is inaccurate in the viscera, which are supplied with an infinitely smaller number of nerve fibres. According to Mackenzie, the situation of the pain of a gastric ulcer, if produced in the ulcer itself, should move when the stomach is caused to move up and down by deep respiratory movements, but it remains stationary. This argument is fallacious, as the brain learns to locate the seat of sensations according to the average position of the part in which they originate, and it is quite impossible for it to recognise changes in position of the part from the character of the sensation, as the impulses which produce this sensation are identical, wherever the part may happen to be at the moment. Thus I have found that when a moveable area of skin is drawn out of its usual position and some part of it is touched or pinched, the sensation produced is referred to the point in space where the stimulated part would normally be, although, if the skin is unusually elastic. this may be several inches away from its actual position. This is exactly analogous to the interesting observation, which Mackenzie regarded as a proof that the viscera are insensitive and that all pain in visceral disease is referred to external tissues: during an operation performed without an anxsthetic the patient felt spasms of pain, which he described as arising near the umbilicus, although they were found to be due to peristaltic waves occurring in a loop of small intestine which had been drawn out of the abdomen and was lying 10 or 12 inches away from the umbilicus.

This "average localisation" of visceral pain explains why it is most accurate in the viscera which move least, such as the gall-bladder, œsophagus, duodenum, ascending and descending colon and rectum, least accurate in the very mobile small intestines, and intermediate in the stomach. If an organ becomes dislocated, pain due to disease in it is still felt in the average position and not in the new position. Thus the pain felt in cases of gastric ulcer is in the same situation whether the stomach is in the normal position or has dropped so low that the lesser curvature may be situated below the umbilicus. In a patient with a painful gallbladder associated with a large liver the pain was felt in the exact situation which the gall-bladder must have occupied before the enlargement of the liver occurred.

Visceral pain often appears to the sufferer to be so deeply situated that it is impossible to believe that it really originates in structures such as the skin and muscles of the abdominal wall, whereas pain produced by disease of these structures feels definitely superficial. Mackenzie answers this argument by pointing out that the pain in herpes zoster may feel as if it were deeply siluated, although, being a result of disease of a posterior root ganglion, it must be a referred pain. But Langley ${ }^{21}$ has shown that the nerve cells of the afferent sympathetic neurons are situated in the posterior root ganglia, inflammation of which can therefore lead to pain referred to the distribution of the sympathetic as well as to that of the ordinary spinal sensory nerves. The

20 J. N. Langley: Brain, vol. xxvi., p. 23, 1903.

21 J. N. Langley in Schifer's Physiology, vol. ii., p. 687, 1900. deeply situated pain in herpes zoster is, in fact, analogous to the abdominal pain, which occurs in disease of the spine or meninges involving the posterior roots of the lower dorsal nerves.

The visceral sensations of fulness and pain probably result from excessive stimulation of the afferent nerves, which normally convey the unfelt afferent impulses concerned in various reflexes. Both Foster ${ }^{22}$ and Sherrington ${ }^{23}$ have pointed out how improbable it is that the viscera should be supplied with pain fibres, which have no other function, for a man may suffer from pain in a particular organ only once in the course of his lifetime. If the pain were conveyed by special nerve fibres it would be difficult to explain how such a special mechanism could have originated, and, when ance originated, how it could have been preserved through perhaps several generations, in which it was never used, in order to give rise to a specific sensation on the one occasion in the individual's lifetime, on which he was attacked by a painful disease of the organ in question.

It is unlikely that the mucous membrane of the alimentary canal contributes to the sensation of fulness or pain, as it is only stretched when the part is greatly dilated, whereas these sensations are felt in the contracted organ when it is subjected to the adequate tension. Moreover, Professor Langley tells me that most of the afferent nerve fibres of the intestines end in the muscular coat, medullated fibres being relatively rare in Meissner's plexus, which supplies the mucous membrane and submucous coat. Possibly the stretching of the connective tissue produced by rapid and extreme distension of the stomach or of a part of the intestine gives rise to a sensation of pain; this would be analogous to the pain, which is apparently produced by tension on the connective tissue capsule of the liver, spleen, and kidneys, when these organs undergo a rapid and considerable increase in volume.

\section{THE MICROBIC FACTOR IN GASTRO- INTESTINAL DISEASE AND ITS TREATMENT.}

BY BERTRAND DAWSON, M.D., B.Sc. LOND., F.R.C.P. LOND.,

PHYSICIAN EXTRAORDINARY TO HIS MAJESTY THE RING; PHYSICIAY TO THE LONDON HOSPITAL; CONSULTING PHYSICIAN TO KING EDWARD VII.'S SANATORIUM, MIDHURST, ETC.

IT is an accepted fact that the stomach is, as regards micro-organisms, the least infected part of the alimentary tract. The organisms which exist there in health are harm. less and in some measure even beneficial. The freedom of the healthy stomach, in contrast to the intestine, from pathogenic organisms is ascribed to the $\mathrm{HCl}$ of the gastric juice. This cannot be a complete explanation. It is more probable that the healthy alimentary canal only permits such microbes as can aid it to get foothold. In the stomach there is not much rôle for bacteria, whereas in the intestine there is.

The freedom of the stomach from germs in good health has led too readily to the assumption that there is the same immunity from pathogenic organisms in ill-health. I think, on the contrary, it can be shown that there are gastric disorders produced by micro-organisms, and that even in patients with structural lesions of the stomach, symptomsit may be contradictory and misleading symptoms-are seen, which are due to secondary infection by bacteria. That such is the fact seems likely, whether we judge from analogy with infections of the intestinal tract or from a study of the gastric conditions themselves.

We constantly have to widen our conceptions of the part played by infections in diseases of the intestinal tract. A few years ago the name "typhoid" was a less exact term than it is to-day, and included several illnesses which we now recognise as distinct. And although paratyphoid, both $A$ and $B$, have been defined, further classification in the future is inevitable. All clinicians meet acute and febrile illnesses of the intestinal tract which they cannot name.

22 M. Foster: Text-book of Physiology, vol. iv., 1891. 1900 
The number of diarrhoea which are shown to be of microbic origin increases, and gastro-enteritis, colitis, and even so-called mucous colitis are often due to microbic infection-. it may be, e.g., by bacterium coli or bacillus acidi lactici (of Hueppe). Cholangitis and cholecystitis owe their origin to infections of the biliary tract, and the former sometimes is an extension from the duodenum. If the bile-duct and the duodenum, why not the stomach?

What is frequently the sequence of events in catarrhal jaundice? A patient previously well feels sluggish, perhaps aches, has a headache, appetite vanishes, tongue is foul and slimy, taste horrible, breath smells. At the same time there are discomfort and distension of the epigastrium. Then after a lapse of two or more days from the commencement of these symptoms, jaundice puts in an appearance. Such an illness is very suggestive of an infection, gastric and duodenal first, and biliary later. Or, again, fever and sore-throat attack a household. In the case of one member the cold "strikes inwardly," to borrow a hallowed phrase. Gastric symptoms follow and even sometimes jaundice. One or more members of a family become feverish and ailing. What are they sickening for? is the anxious inquiry. A day or two later some gastro-intestinal symptoms develop. With a feeling of relief the trouble is pronounced to be "stomach." Such an illness suggests a bacterial origin.

Turning to chronic ailments, the following is a picture by no means rare. The patient is constantly inert and "livery." His mouth is sticky, the mucous membrane of the lips glazed, tongue slimy, pharynx shows a ropy mucus and looks sodden. Every few days he describes himself as "rotten." His discomfort, though not localised to, is accentuated in, the upper abdomen. Such people never have a clean tongue, feel seedy for weeks at a time, eat because they are exhausted not because they are hungry, wake in the morning heavy and wretched. Few realise what an effort life in any active sense is for them. If they be people of strong wills, they drive themselves through life carrying their burden, but many find the struggle too much, let their abdomens dominate their lives, and sink into inactivity and despondency. Or, changing the picture in detail, unpleasant tastes, offensive breath, flatulence, gastric discomfort, may be prominent features: Gas is expelled from the stomach whether fasting or after food, and may be offensive. Where lies the cause?

It may be suggested that the offensive smell originates from septic gums or nose, but it exists in cases where the mouth and nose are normal. Moreover, the gas is detected on passing the tube into the fasting stomach, and it is reasonable to conclude that in such cases the gas and its offensive attribute originate in the stomach. Retained stagnant contents and unwise eating and drinking are no doubt frequent causes, but in the group of cases to which I refer there is no retention of contents, and the symptoms persist in spite of careful dieting and a reasonably regulated life. An infection of the gastric mucous membrane offers an explanation of the facts. Nor is it by analogy improbable. Inflammations of mucous membranes in other parts of the body result from microbic infection. One may instance cystitis, cholecystitis, bronchitis. We are no longer content with a diagnosis of bronchitis, nor do we regard chill as a sufficient cause. We examine the sputum not only to know that tabercle bacilli are absent, but what organisms are present. The same holds true of gastritis.

Gastro-intestinal infection is no new idea. It has been dealt with by many workers, and with especial clearness by Herter. I venture to think that its existence has not been sufficiently recognised as regards the upper regions of the alimentary tract. That the gastric mucous membrane can occasionally be the seat of acute infection is well established. One may instance the severe gastritis, with or without hæmatemesis, which occurs during the course and often near the end of acute streptococcal or pneumococcal septicæmias. And there are, again, the rare cases of membranous gastritis due to diphtheria. If acute infections exist, why not those of lower grade running a more chronic course and producing their effects slowly, the manifestations being sometimes gastro-intestinal, and at other times more general, taking the form of failing energy, anæmia, \&c ?' No doubt gastric manifestations need not presuppose gastric infection. The bacteria may be elsewhere. It may be, e.g., the intestine or the gums, and the gastric disturbances result from the toxins being excreted by the gastric mucous membrane. It is admittedly difficult to differentiate in practice between an intoxication and a septicæmia. The gastritis which goes with oral sepsis may be toxic or a secundary infection of the stomach. The dyspepsia of anæmic women, associated as it occasionally is with hæmatemesis, probably falls into the same category.

With a view to determining the presence and significance of micro-organisms in the stomach, the investigation described below has been adopted. In order to make the results as

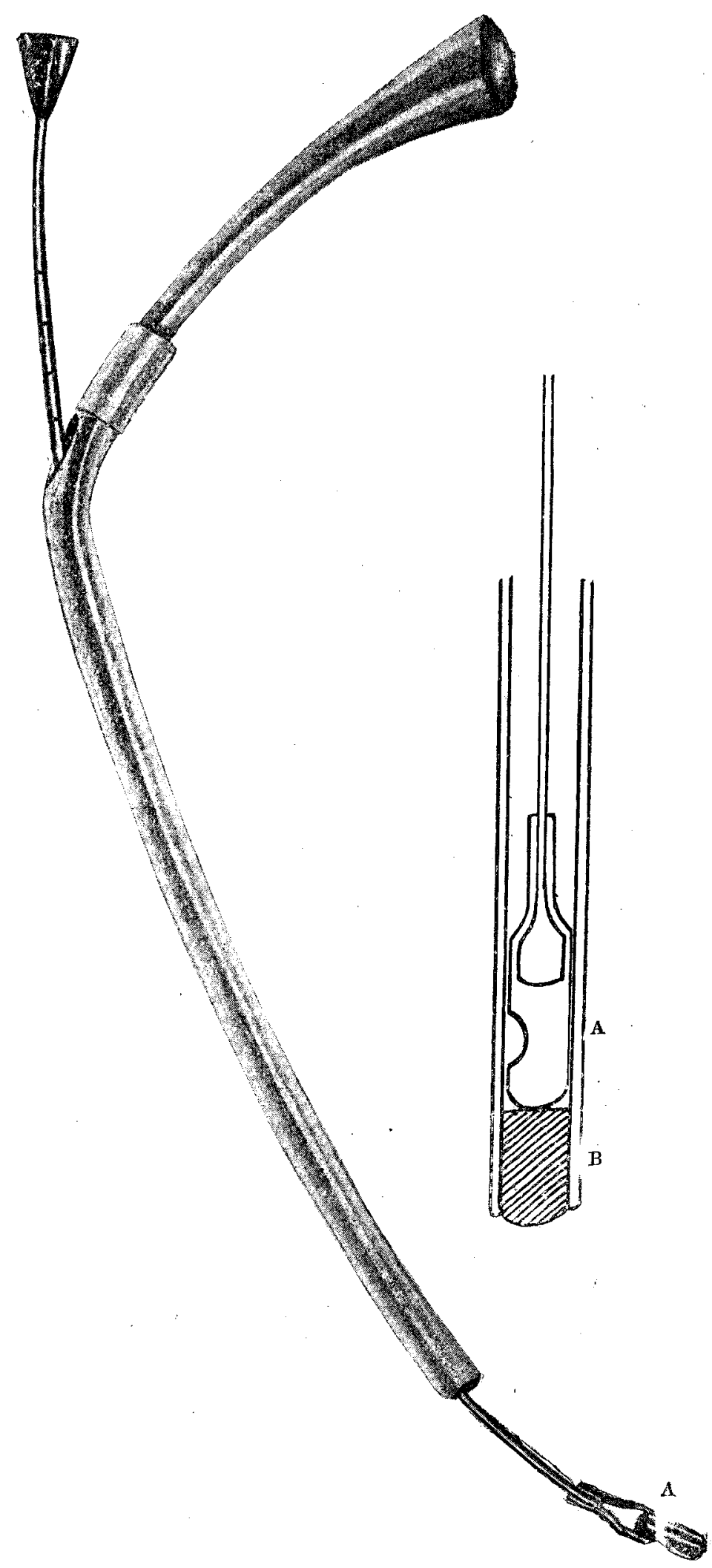

Instrument used to obtain sample of stomach contents. A, Bucket. B, Cotton-wool or potato.

reliable as possible, I at first selected cases with definite features, such as furred tongue, foul breath, offensive smell. Care has been taken not to select patients with dilated stomachs, bad teeth, septic gums, adenoids, those who do not eat and drink temperately, and those who are habitually constipated. Hospital patients have not been chosen because their mode of life is seldom sufficiently careful and regular. The patient to be investigated is on ordinary, but light, solid diet. The instrument is usually passed in the early morning into the fasting stomach, no food or drink having been taken 
for 10 or 11 hours previously. The mouth is first washed. The procedure consists in abstracting a sample from the fasting stomach contents, without that sample being contaminated. The outer covering of the instrument is indiarubber, resembling a stomach-tube without lateral openings. Inside this is a curved steel ribbon, on the end of which is a small bucket. Before use the two parts are sterilised and the steel band inserted into the rubber tube, so that the bucket is an inch off the opening. The last inch of the tube is plugged with either sterilised cotton-wool or potato. The tube is passed in such a way that the concavity of its curve looks 'to the patient's right. After it has entered the stomach the bucket is extruded beyond the tube, and then after a moment drawn back well under its cover and the tube withdrawn from the stomach. With practice the tube and bucket can be made to travel towards the pylorus, which it is an advantage to secure.

The contents of the bucket are dealt with as follows:Loopfuls are remored from the bucket and placed on the various media, and grown at a temperature of $37^{\circ} \mathrm{C}$. Smears are also made and examined after Gram's method of staining. The following media were used:(1) bouillon; (2) agar ; (3) glycerine agar ; (4) acetic acid agar; (5) lactose bile agar ; (6) blood agar or serum agar ; (7) oleic acid and neutral red. Hydrochloric acid agar was also experimented with, but was found unworkable, for although it set the surface was immediately broken up by the platinum needle.

Acetio acid agar.-By using this medium it was often found possible to inhibit the growth of many organisms of the staphylococcic groups, of moulds and sarcinæ, whilst some of the streptococci were found growing in pure culture.

Glyoerine agar was found to be one of the best media for the cultivation and differentiation of the streptococcal groups, and was used as the medium on which growths were made for vaccine work. When organisms of the B. coli group 'were found, in order to prevent overgrowth and for differentiation from cocci growth under anaerobic conditions was adopted. Anaerobosis was also used to keep gas-forming organisms alive.

Many gastric and intestinal contents have been thus examined. The bacteriology has been done by Dr. John Matthews and Dr. J. E. Adler, partly at Sir Almroth Wright's laboratory at St. Mary's Hospital, and partly at Dr. W. Bulloch's laboratory at the London Hospital, and I should like to express my indebtedness for their valuable aid. 'To Dr. Adler my grateful acknowledgments are also due for much able help in working out the cases. The cases examined comprise 38 cases of disorders of the gastro-intestinal and biliary tracts. Of these, 13 were sterile, 16 gave definitely positive results, while in 9 the organisms were a medley and only few in number. The organisms found in the stomach smears and cultures were streptococci, diplococci, bacterium coli, bacillus acidi lactici of Hueppe, and (in one or two (cases) staphylococci. Cultures were often taken from the tongue and naso-pharynx at the same time. In some cases analyses of the stomach contents and of the urine were made. If there were intestinal symptoms the fæces were analysed. In these analyses I have several times had the help of Dr. P. J. Cammidge's experience. In a few cases the hydrochloric acid content was very low, but in most the percentage of free and active hydrochloric acid together varied between 0.14 and 0.28 per cent.

A. Lockhart Gillespie (1) in a laborious research investigated the food contents in numerous patients. The rubber stomachtube wsed was washed first with boiled water, and then with - alcohol. The first 20-30 c.c. of the stomach contents were allowed to escape, then a small quantity was caught in a : sterilised test-tube. Gillespie enumerates 24 micro-organisms thris found from the contents of the stomach. Only a few in the list are, however, pathogenic. In using food contents the diffliculties must have been greater than in using fasting sontents.

Hirschberg and Liefmann (2), with the same object in view, uave adopted the following method. The patient received as an evening meal a litre of milk and two eggs at night. He then fasted till the morning, when the stomach was washed ont with 200 c.c. of sterile saline solution. A sterilised stomach-tube and funnel were used, the operator's thands being covered with gloves. The fluid was composed af shreds of mucus, but no food particles were found. "The mouth of the patient was washed out with peroxide of bydrogen to get rid of the saliva. A little of the saliva was collected for bacteriological examination in a sterile glass, and afterwards plated out in 4 dilutions, 1-10, 1-100, 1-1000, 1-10,000. The stomach washings were also plated out in the following strengths: 1-10,1-100, 1-1000, 1-10,000 1 c.c. of each being inoculated into alkaline agar and acid "Vierwurze" agar, and then being poured out into Petri's dishes. The authors give their results in 40 cases, 29 representing gastro-intestinal disorders, and 11 various other diseases. The stomach contents were sterile in 14 cases, of which 6 were gastric and 8 non-gastric disorders. The organisms found by Hirschberg and Liefmann comprised streptococci, diplococci, staphylococci, B. coli, B. para-coli. Comparison between Hirschberg and Liefmann's results and mine is interesting from the fact that my investigations were begun before their paper appeared and were advanced before I had the pleasure of reading it.

Hydroohloric Aoid Content and Bacteria in the Stomach.

There seems no doubt that hydrochloric acid, especially in the free form, restrains bacterial growth. Gillespie's investigations, confirming those of MacFayden (3), show this. But in the living stomach it is above and beyond the free hydro chloric acid a question of specific resisting power. When the stomach is healthy it is free from microbic infection because its resisting power is high, not only because free $\mathrm{HCl}$ is present.

My cases show that pathogenic organisms can exist in the stomach with a normal or raised hydrochloric acid content. Herter (4), too, refers to cases of putrefaction going hand in hand with hyperchlorhydria. Thus the inhibiting effect of hydrochloric acid on microbic growth in the stomach has its limitations, and confirmation is given to the view of Cushing and Livingood (5) that there are influences outsid? the bactericidal power of the gastric juice to account for the amicrobism of the healthy stomach. Hirschberg and Liefmann concluded that sterile contents go with normal secretion and motility, and if acidity or motility fail bacteria make their appearance. I would modify their conclusions by saying that failure of acidity and motility is primarily evidence of lower resisting power to invasion. Such failure is not necessarily the direct cause of the invasion, but rather its concomitant.

An interesting point which has come out in these investigations is the frequency with which the fasting contents are sterile in gastric and duodenal ulcer, provided there is no marked dilatation. So often is this the case that I begin to regard it as a diagnostic point worthy of attention. It is interesting why sterile contents should go with ulcer; it would not have been surprising if the opposite had been the case. That the sterility is not alone due to the raised hydrochloric acid content has been already shown. All the cases referred to have been proved at operation. In Hirschberg's and Liefmann's list of cases there are three gastric ulcers, and in all, their gastric contents were sterile. Of seven cases recently under my care for ulcer, and all confirmed at operation, six showed sterile contents, whereas in the one which did not there was dilatation due to pyloric stenosis.

CASE 1.-Man, aged 38, ailing for two years ; constant epigastric distress, tongue always furred, nasty taste, fœit breath, offensive eructations from stomach, anæmia, muddy complexion, restless, some loss of flesh, no suppuration of gums, no constipation, no undue retention of stomach contents. Test meal showed 0.15 per cent. free hydrochloric acid. The bucket content showed sometimes a diplococcus and sometimes a streptococcus in short chains. Cultures grew this diplo-streptococcus almost pure. In broth the organism grew in short chains. When first grown on broth, and to a less extent on agar, it yielded an offensive gas resembling in odour the patient's eructations, though it gradually lost this power in subsequent subcultures under aerobic conditions. The gas contained carbonic acid, but owing to imperfect methods not enough was obtained for complete analysis. In this patient a similar gas-producing organism was obtained from the naso-pharynx, which, though a little boggy and red, was not discharging pus.

CASE 2.-This question of gas production is further illustrated by the following. The patient, a man about 40 , had epigastric discomfort, dirty tongue, eructations like the last case, but less severely and less constantly. His chief complaint was attacks of pain which in every way resembled gall colic. He had never had jaundice. My diagnosis was cholecystitis with gall-stones. His stomach was bacteriologically examined and a diplo-streptococcus grew freely, as 
in Case 1. This organism produced gas of the same foetor, and by putting the cultures under coal-gas the production was free. An analysis of this gas showed it to consist of carbonic acid, and the fœtor to be due to indol and skatol. There had clearly been decomposition of proteid in the stomach, as the result of bacterial action. The carbonic acid gas only harmed by producing discomfort and interfering with stomach motility. It was the products of proteid cleavage which caused the trouble. Possibly no more occurred in these stomachs than is habitual in the ileum, but the latter is provided with means and devices for dealing with putrefactive processes, whereas the stomach is not.

These two cases have been set forth together because they presented examples of gas-producing organisms. They had not a close clinical resemblance to each other. Case 1 I regarded as a chronic gastric infection, and I may say in passing that striking benefit followed vaccine therapy, where all other methods failed. Case 2, as already stated, was regarded by my surgical colleague, Sir Frederick Eve, and myself as gall-stones, and operation was decided upon. Shortly before the operation I thought it would make for completeness if a bacteriological examination of the stomach was added to the analyses of test-meals, urine, and frces.

At the operation the gall-bladder and bile-ducts were carefully examined; the former was emptied and no gallstones found. There was no evidence of ulceration of stomach or duodenum. As the attacks of pain were a serious matter to the patient it was thought wise to examine the appendix. This was found somewhat shrunken, with some old adhesion round it, but nothing to suggest any recent mischief, or any causal relationship between it and the symptoms. As the appendix was exposed it was wisely removed. As nothing was found in the abdomen to explain the symptoms the patient was injected with his own vaccine during a period of convalescence. Although he is quite well, it would be manifestly unfair to draw too confident conclusions from the vaccine treatment. The question for reflection is, What was the cause of the patient's pain? Was it a pyloric spasm? Probably yes, the spasm being produced by gastric irritation, and this in turn, perhaps, resulting from gastric infection. I have met similar cases in which the manifestations have been similariy definite, and yet a blank has been drawn at the operation in spite of all structural causes having been searched for.

$$
\text { The Appendix and Gastric Disturbance. }
$$

A further reflection arises in connexion with Case 2. What is the cause of the undoubted gastric manifestations which sometimes are associated with a chronically diseased appendix? Are not these cases secondary gastric infections? In some instances the removal of the appendix is certainly followed by subsidence of the gastric symptoms. It is equally true that in other cases the hoped-for cure does not follow the operation, and the gastric symptoms persist as before.

The position at present is too much, "If not gastric or duodenal ulcer, if not gall-stones, then it must be appendix." The latter is sometimes responsible for a sense of disappointment at operation, in that it does not show morbid change sufficient to account for the symptoms laid to its charge, and this disappointment is apt to seek relief in the unconscions exaggeration of insignificant structural changes. It is possible, however, that an appendix, without showing any definite structural change, may still be a focus of infection, and that, by its removal, gastric and colonic symptoms will subside. Unfortunately, it not infrequently occurs that the appendix is not the focus, but shares in an iniection of the cxcum, and by its removal the mischief is not touched. Oloser examination of the colon, clinical and bacteriological, is diminishing these mistakes.

$$
\text { Infections of the Biliary Tract. }
$$

Case 2 also suggests the inquiry: Can there exist disease of the biliary tract simulating gall-stones? The answer is, in my judgment, in the affirmative. Gall-stones are the structural end-product of a tedious infection. They are the common, but not the only, result of such infection. The following case illustrates this contention well :-

CASE 3.-A lady of middle life for many months had had a constant ache and discomfort in the right upper abdomen, going through to the back. From time to time the pain became more acute and of the nature of gall-colic. Without going into further details, the diagnosis arrived at was gallstones with duodenal ulcer as a possible alternative, and this opinion was shared by Mr. J. Bland-Sutton. At the opera. tion the gall-bladder was bound down by lymph and recent adhesions, most of which could be detached easily by the finger; the serous surface of the gall-bladder was the seat of a subacute inflammation. A careful examination was made of the abdomen, and nothing else wrong was apparent. The gall-bladder was opened, and it and the ducts were carefully examined, and these were normal, with no sign of gall-stones, past or present. A portion of the gall-bladder wall and the contents of the latter were subsequently examined bacteriologically. The bile was sterile, but the wall, which included the serous coat, grew a streptococcus growing in short chains.

This patient's symptoms are now easily explained. The subacute pericholecystitis caused the constant ache, and when the gall-bladder tried to contract to empty its contents at certain stages of digestion the colicky pain was produced. An interesting question is, Would this patient, if left, ever have got gall-stones?

\section{Colitis.}

I now come to the relation which, I think, sometimes exists beween colitis and the upper portion of the alimentary tract. When the discussion on colitis was being held at the Royal Society of Medicine in 1909, I put forward the view that colitis was looked upon as too strictly a disease of the colon only, whereas there were reasons for thinking that sometimes the colon manifestations are the final expression of a disturbance which has commenced in the upper part of the alimentary tract, just as the digestive changes in any given part of the alimentary canal are influenced by the changes which have occurred in the portions above it. The reasons given for this view were as follows. A patient with chronic colitis is liable to relapses, and these relapses can often be observed to commence with gastric disturbance, and the more definite colonic symptoms to follow, it may be some hours or a day later. If the fæces of colitis patients are examined, they will sometimes show deficient fat digestion. The urine will sometimes give a marked indican reaction, pointing to trouble in the small intestine, and in other instances Cammidge's pancreatic reaction is observed in the urine. (This would be a convenient place to make reference to Cammidge's pancreatic reaction. Differences of opinion exist as to its reliability. Some regard it as useless. This, I think, is because they judge it by too high a standard. My conclusion, based now on many observations, is that it is a good test, well worth consideration in conjunction with other evidence, but that like other data-both clinical and bacteriological -it has its limitations and will sometimes lead one astray.)

The investigations which form the subject of this paper support the above view. In the example I am about to describe, the colon and stomach were separately investigated, the examination of the colon contents by Dr. Matthews pointed to $B$. acidi lactici of Hueppe being the guilty microbe ; the examination of the stomach by Dr. Adler, and subsequently confirmed by Dr. Matthews, showed B. acidi lactici of Hueppe, a diplococcus, and a Gram-positive streptococcus. The finding of the first of these organisms in both colon and stomach was most interesting and suggestive. But this is not all. The patient, who was a severe and longstanding example of colitis, and had been thoroughly and variously treated, improved slowly with vaccine of $B$. acidi lactici, but after some months the improvement became arrested. A vaccine of the stomach streptococcus was then added to the former, and the improvement recommenced and at increased rate; and the patient to-day, on the testimony of herself and friends, is in better health than for many years past.

CASE 4.-The above case is worth setting forth more fully. Duration, on and off for ten or more years; the usual abdominal symptoms and signs, including the distended and spent cæcum and ascending colon, contrasting with the cordlike descending colon; at the commencement of the exacerbations the stomach would become distended; tongue frequently dirty and mouth unpleasant, nutrition poor, low weight, sallow complexion, easily fatigued; frequently feeling ill; abdominal discomfort with recurrences of pain along the colon; discharge of mucus. There was a history of jaundice eight years ago without pain, lasting three months. Fæces semi-solid, mixed with mucus and mucous casts; well-marked reaction for stercobilin; marked excess of unabsorbed fat, 40.9 per cent. of dry weight as against 
normal 15-25 per cent. ; high proportion of unsaponified fat, 26 per cent., as against 10-15 per cent. Bacteriologically, two varieties of colonies on lactose litmus plates, which their respective sugar reactions put into two groups: (1) B. acidi lactici of Hueppe, which gave an index of 0.41 ; (2) Friedländer, which gave an index of 1.08. Urine showed marked indican and pancreatic reactions. The stomach was bacteriologically examined, and, as stated above, B. acidi lactici, a diplococcus, and a Gram-positive streptococcus were found.

This patient was treated with a vaccine, first of $B$. acidi lactici, commencing with a dose of 6 millions. Later the mixed vaccine of $B$. acidi lactici and the stomach streptococcus was given, the dose reaching 30 millions of the former and 20 millions of the latter. This patient has slowly improved, and, on the testimony of herself and friends, is better than for years past. The improvement is testified by increased weight, more healthy colour, a feeling of energy and well-being to which she was a stranger; gastric digestion stronger; mucus-with the exception of occasional and slight recurrences-banished from the stools. This patient is still under treatment, and is still improving. There has been no drug treatment, but the diet is carefully selected, and there is a watchful avoidance of fatigue, for let such of these patients who are of the spare type over-exert, and the whole digestive tract will go wrong forthwith.

Whatever conclusions are drawn, the observations in the above case are, I venture to think, interesting and suggestive. For myself, I do not think bacterial infection is the only cause of colitis. It is rather a direct causal factor of varying import, in some cases more dominating, and in others less dominating.

CASE 5 illustrates again the relationship between the lower and upper alimentary tract. The patient, aged 30 years, led an active out-of-door life as a farmer. He had manifestations of colitis; discomfort and dull pain in the large intestine, especially over the cæcum which was dilated; passed mucus with the stools; was slightly constipated. In addition to the foregoing, his tongue was constantly furred, mouth unpleasant, and his breath was offensive, to the distress of his wife teeth and gums healthy; stomach distended. No bacteriological examination was made of the stools, and the ordinary treatment for colitis was adopted. The colon manifestations improved, the abdominal discomfort diminished, the mucus was less, and the bowels more regular. But mouth and stomach symptoms persisted.

The stomach was then examined bacteriologically, and a diplococcus found and cultivated. Vaccine treatment was alone adopted. At first after small doses of 20 millions the improvement was transitory and sometimes doubtful. When the dose was made 30 and 40 millions at 10-14 davs intervals

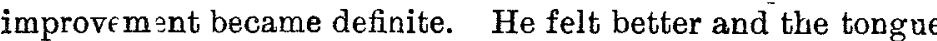
was cleaner, and his wife bore testimony to the breath losing its offensiveness. In the first three months of improvement, the symptoms began to return when the next dose was due, but later he became quite well and remained so. On two occasions the symptoms were aggravated for 24 hours after the injection, presumably negative phases.

CASE 6.- Patient resembled in sex, age, and general clinical aspect Case 4. There was in addition some visceroptosis. The manifestations were both gastric and intestinal. The features of the urine were well-marked indican reaction, a negative pancreatic reaction, and suspended calcium-oxalate crystals; nrea and phosphates were normal; chlorides subnormal. The freces were acid, the reaction for stercobilin subnormal, total fat was high, and the excess was nearly all unsaponified, suggesting diminished pancreatic function. This patient's stool, though for the most part of normal colour, showed pale almost white patches, like the claycolonred stool of jaundice Dr. Cammidge reported these patches to consist of " masses of combined and free fatty acids, chiefly the former, mixed with bacteria, mostly Grampositive." A vaccine of the patient's $B$. coli was used in combination with other treatment, as in Case 4, and with the same satisfactiory results.

CASE 7. - The following case is suggestive. A woman, aged 35 years, tall, with shallow abdomen, leading a rational life in the country, for years lacked strength, was easily tired, and constantly had spells of abdominal discomfort. This latter took the form either (1) of a dull ache more on the right side, due to a prolapsed mobile right kidney, or (2) pain referred to mid or right epigastrium from a half to one hour after food. No vomiting, no hunger-pain, no localised tenderness; but foul tongue, unpleasant taste, stomach flatulence, intermittent. headache. The clinical picture is common enough. Amongst other causes, tea-drinking would produce it, and so would imperfect evacuation of the colon. But no such causes existed ; the symptoms persisted in spite of careful dieting and medivaments and a strong desire to be better. Why should it be thus, was the problem constantly pressing for answer.

Further investigation was undertaken. The analysis of stomach contents after the usual test-meal of toast and tea showed as follows :-Well-marked reaction of free $\mathrm{HCl}$, the percentage being 0.12 ; physiologically active $\mathrm{HCl}, 0.16$; organic acids, 0.02 per cent. ; a marked reaction for dextrin and a fair reaction for sugar ; only a trace of lactic acid.

This analysis rather suggested a diagnosis of hyperchlorhydria, and it might be argued that the cause lay in the mobility of the kidney. But the stomach symptoms persisted. when the kidney symptoms had disappeared, and such a view does not explain the following further data. The fæces and urine were examined by Dr. Cammidge. The urine gave a well-marked reaction for indican, a trace of urobilin, and calcium-oxalate crystals in the microscopic residue, and a positive pancreatic reaction. The fæces, though normal in appearance, showed a strong alkaline reaction, a wellmarked reaction for stercobilin, and an excess of unabsorbed fat, the excess being mainly in the saponified fat-viz, $19 \cdot 5$. per cent. of the dry weight as against a normal of 10-15 per cent.

These observations, both clinical and chemical, pointed to the existence of a chronic inflammation, an infection of the duodenum and upper intestine with pancreatitis and recurrent gastritis. It might be suggested that pancreatitis was the bottom of the trouble, but is it not probable that pancreatitis itself is a low-grade infection spreading from the intestinal tract? Assuming this patient does not remain. better, one foresees various possible developments; the trouble may focus itself in the stomach, and chronic catarrh with progressive atonic dilatation and gastroptosis result; or again, the infection of the upper intestine may derange the lower, and mucous colitis may result ; or again, the infection may extend into the biliary tract and cholelithiasis eventuate. No doubt the order may be reversed, and inflammation of the appendix and colon secondarily involve stomach or duodenum. It is within the experience of all clinicians how despairing such cases as the one just described can be from the point of view of treatment, and this one defied effort.

The stomach was then bacteriologically examined and a diplococcus was obtained in culture, and from this a vaccine was made. The dosage began with 20 millions and gradually rose to 80 millions. Marked improvement followed. The patient (an intelligent critic) asked that the injections might. be continued. Whether this is a case of post-or propter$h o c$, it is impossible to be confident. It is a more difficult result to judge fairly of than where objective signs, like foul eructations, exist. The patient's improved health has persisted for many months, and is better than for years past.

The Mouth and Pharynx.

The ill-effects of septic gums are not being considered in this paper. They have received able consideration from many writers, and no clinician can fail to be convinced how far-reaching they are.

Apart from the gums there appear to be infections of the mouth and pharynx which have relationship to gastrointestinal derangement. How often it happens that throat symptoms are precursors of gastro-intestinal ones! Many dyspeptics have chronic pharyngitis. In many, no doubt, errors of diet or habit account for the double condition When such errors are absent the trouble is often called gouty, though not always on sufficient grounds. Some of these cases will be found to be chronic infections-either of the pharynx or of the stomach or both. In two at least of my cases associated gastric and pharyngeal signs improved together from vaccine treatment.

CASE 8.-The following is a further example: A man, otherwise healthy, was liable to recurrent attacks of pharyngitis. With them he would get dyspeptic symptoms what he called "bilious" attacks, and on two occasions calarrhal jaundice supervened. Later, for his throat trouble, he fell into the hands of a vaccine therapist who discovered his pharyngitis was due to pneumococcal infection. Under the vaccine treatment employed, not only the pharyngitis 
disappeared, but the digestive trouble at the same time. It cannot be asserted there was gastric infection here, for the stomach was not investigated, but the digestive derangement was too widespread to have been produced by swallowed discharges, which undoubtedly produce gastric catarrh. In a case quoted above the same gas-producing organism was alike found in the stomach and pharynx.

Another familiar clinical experience is the patient who seldom or never is without a furred tongue. When at his best there is nothing but the tongue; when not at his best there are associated dyspeptic symptoms. I only refer to cases that are intractable in spite of careful living. The following case is an interesting illustration.

CASE 9.-The patient was an active and otherwise healthy man. The condition of his tongue and digestion were as above described. His fasting stomach contents were practically sterile, but his tongue yielded a rich growth of a long streptococcus, to which his blood gave a low index. A vaccine was made and tried, and the result was striking improvement lasting many months. The trouble recurred, and the patient was so convinced of the previous benefit that he requested a repetition of the treatment, which his medical man carried out, with like beneficial results.

There is likewise relationship between the pharynx and the intestine. Recently I have had an example of recurrent attacks of pharyngitis occurring in association with recurrent mucous colitis. The pharyngitis was due to pneumococcus, and a vaccine prepared from this pneumococcus improved both conditions, and when suitable doses were exceeded both conditions were temporarily aggravated.

I do not suggest that the colitis was also a pneumococcal infection. What is more probable is that the lowering of resisting power to pneumococcus in the throat leads to a correlated lowering of resisting power in the colon to some organism (say, B. coli or B. acidi lactici (Hueppe)) already there. This correlation of resisting power to different organisms is important. The invasion of a region by one organism seems to carry with it a lowering of resisting power to other organisms, and not necessarily limited to the same region. This suggestion affords an explanation of many clinical findings. A parallel may be found in lead poisoning, which undoubtedly produces a greatly increased liability to gout.

\section{Tonsillitis and Appendicitis.}

The above remarks remind me of a saying more than 20 years ago by my revered chief, $H$. G. Sutton-not only one of the greatest clinicians of the nineteenth century but one possessed of that rarest of gifts-insight. He told me one day that tonsillitis and appendicitis find their home in the same subject. The truth of this was not appsrent to me then, but Sutton's sayings have a wonderful knack of showing themselves true with the unfolding of the years, and I have satisfied myself there is sound clinical evidence for the exist. ence of this association between inflammations of the tonsils and appendix.

Not only is the sequence of the two events met with, but removal of an appendix will sometimes cure a chronic throat. It could hardly be said that treatment of the throat would cure an appendix once lighted up, though if the above relationship exists a much greater importance must be attached to the state of tonsils in their relation to the prevention of appendicitis. Tonsils need to be judged not only by their size and quantity but by their quality; those of moderate size are sometimes very septic, and as such are sources of danger.

In tinis connexion it is interesting to note that Hasse (9) has recorded the occurrence in an institution within two months of 30 cases of appendicitis and mostly following tonsillitis. Again, Hans Bachhammer (10) has reported eight cases of suppurative tonsillitis in concurrence with appendicitis. Similar cases have been reported by Apolant in 1897, Jessen in 1898, and Weber in 1902.

Returning to the gastric aspect: broadly speaking there are three pictures presented by the bacteriological examina. tion of the contents of the fasting stomach :-1. The contents are quite or almost sterile. This is the condition of the healthy stomach 10-12 hours after a meal. It is also the condition often found in cases of ulcer. 2. Some organism like a diplococcus or streptococcus is found in great predominance, sometimes almost pure. 3. A medley of organisms is found, denoting a stomach of low resistance, but not necessarily associated with any clearly defined group of symptoms. Sometimes these organisms suggest contamination from above.

That pathogenic organisms exist in the stomach, and even sometimes in spite of the HCl being normal, or in excess, is I think clear. That these organisms have actual foothold and are not mere incidental importations is more difficult to establish. That such foothold, such infection, does occur is supported by the following considerations:-It is in accordance with what we know obtains in other portions of the alimentary tract and the biliary canal. It explains the facts of certain gastro-intestinal disorders. The isolated organisms reproduced the gas which had formed in the stomach. Vaccines prepared effected definite improvements and cures. In some instances accidental over-doses temporarily exaggerated the symptoms, and the production of a negative phase points to the causal organism having been obtained.

The route of invasion no doubt varies ; it may be from the cosophagus, duodenum, or blood stream. In some cases the biliary canaliculi may be infected by the blood stream, and then follow descending cholangitis, duodenitis, gastritis. This is what must occur in those acute infections beginning, say, with fever and tonsillitis and passing on to jaundice and gastric disturbance. Sometimes the sequence would seem to be reversed, and a chronic low-grade infection of stomach and duodenum may extend, producing intestinal or biliary derangement. Are not thus explained those cases of cholelithiasis which have for years gastric symptoms as their sole manifestations? Why does Carlsbad undoubtedly do cholelithiasis patients good? Not assuredly because it affects the gall-stones already there, but because it affects beneficially the gastro-duodenal mucous membrane (the chronic infection).

As regards vaccine treatment, in some cases its effect was very convincing. The patients, judged both from the subjective and objective standpoints, improved or were cured. Some relapsed after an interval without treatment, and would come back asking to be infected again, and would again improve. On the other hand, there were instances in which vaccines entirely failed. This discrepancy in results is especially noticeable in colitis-some cases benefiting and others remaining untouched. The larger the neurasthenic element in a case, the less favourable the prospect for vaccine treatment.

Just as the foregoing remarks seek to show that infection is a factor amongst others to be considered in gastro-intestinal diseases, so vaccine therapy takes its place for consideration beside other treatment. No doubt careful eating and drinking, less worry and work, more fresh air and exercise, are potent factors in curing digestive derangements. But such lines of treatment are not always sufficient even if they are feasible.

In conclusion, I am conscious that there are gaps in the evidence and that the investigations have not always been complete. The latter have been conditioned by the tolerance and elusiveness of patients and the exigencies of routine work. I venture to hope however that they justify interest and further inquiry.

Bibliography.-1. Gillespie, A. Lockhart: Bacteria of the Stomach Journal of Pathology and Bacteriology, Fidinburgh and London," 1892-93, pp. 279, 302. 2. Hirschberg and Liefmann : Zur Bacteriologie des Magens, Berliner Klinische Wocherschrift, 1909, Band xlvi., pp. 1407-11. 3. MacFadyen, Allen: The Behaviour of Bacteria in the Digestive Tract, Journal of Anatomy and Physiology, vol. xxi., p. 227, 1887. 4. Herter, C. A. : Lectures on Chemical Pathology Surgical Notes upon the Bacteriology of the Upper Portion of the Surgical Notes upon the Bacteriology of the Upper Portion of the Alimentary Canal, Johns Hopkins Hospital Reports, vol. ix., 1902, p. 543. 6. Capitan and Moran: Recherches sur les Micro-Organismes de l'Estomac, Comptes Rendus (Hebd.) de la Société de Biologie, Séance, Jan. 12th, 1889, p. 26.7 Hale White and Eyre, J. W.: Tho Results of a Year's Use of Vaccines in General Medicine, Proceeding of the Royal Soeiety of Medicine, Therapeutical and Pharmacological Section, p. 149, May 4th, 1909, vol. xi., part 3. 8. Herter, C.A. : Th Comm Bacterial Infections of the Digestive Tract and he intoxications acising from them, 1907. 9. Hasse: Ueber eine Sindemie von Appendizitis, Munchener Medizinische Wochenschrift, 1910, p. 1123 10. Bachhammer, Hans : Einiges über Tonsillitis und ihre Beziehungen
zu anderen Frkrankungen, Archiv fur Laryngologie, 1910, Band xxii., 332

Wimpole-street, W.

OwING to the indisposition of H.R H. the Duke of Connaught the opening of the Research Institute of the Cancer Hospital (Free), Fulham-road, S.W., announced for Thursday, April 27th, has been postponed. 